\title{
Icriodus riosi, NUEVA ESPECIE DE CONODONTO EN EL DEVÓNICO INFERIOR DEL PIRINEO CENTRAL ESPAÑOL
}

\author{
Carlos MARTÍNEZ-PÉREZ \\ Departamento de Geología, Universitat de València, C/ Doctor Moliner, 50. \\ 46100-Burjassot (Valencia, España). Carlos.Martinez-Perez@uv.es
}

\begin{abstract}
Martínez-Pérez, C. 2010. Icriodus riosi, nueva especie de conodonto en el Devónico Inferior del Pirineo Central español. [Icriodus riosi, a new conodont species from the Lower Devonian of the Spanish Central Pyrenees.] Revista Española de Paleontología, 25 (2), 139-147. ISSN 0213-6937.
\end{abstract}

\begin{abstract}
The conodont Icriodus riosi n. sp. from the Lower Devonian of the Spanish Central Pyrenees is described and discussed. It is characterized by a spindle with straight margins and a short outer process that forms an obtuse angle with the main axis of the element and it is probably closely related to the curvicauda-celtibericus group. It ranges from the pireneae Zone (late Pragian), slightly below the kitabicus Zone, to the early part of the nothoperbonus Zone (late early Emsian). The abundance and diversity of the different species of the genus found in the same sections, e.g. Ic. curvicadua, Ic. celtibericus, Ic. bilatericrescens or Ic. gracilis, suggests that during this time interval an evolutionary radiation of the group took place, a phenomenon that until now had only been documented for the genus Polygnathus.
\end{abstract}

Keywords: Conodonta, Icriodus riosi n. sp., Lower Devonian, Spanish Central Pyrenees.

\section{RESUMEN}

Se describe y discute el conodonto Icriodus riosi n. sp. del Devónico Inferior de los Pirineos Centrales Españoles. Esta especie se caracteriza por presentar un cuerpo principal con bordes rectos y un corto proceso posterior externo que forma un ángulo obtuso con el eje principal del elemento, estando ésta probablemente emparentada con las especies del grupo curvicauda-celtibericus. Su rango estratigráfico abarca desde la Zona pireneae (Praguiense tardío), ligeramente por debajo del comienzo de la Zona kitabicus, hasta la parte inferior de la Zona nothoperbonus (Emsiense inferior tardío). La abundante y diversificada asociación de diferentes especies del género en las mismas secciones, como por ejemplo Ic. curvicadua, Ic. celtibericus, Ic. bilatericrescens o Ic. gracilis, sugieren que durante este intervalo temporal se produjo un proceso de radiación evolutiva del grupo, fenómeno que hasta ahora sólo había sido documentado en el género Polygnathus.

Palabras clave: Conodontos, Icriodus riosi n. sp., Devónico Inferior, Pirineos Centrales Españoles.

\section{INTRODUCCIÓN}

Los Pirineos representan actualmente una de las pocas regiones del mundo con grandes posibilidades para la bioestratigrafía del Devónico que queda por investigar en profundidad, siendo ciertamente una de las áreas clave para el establecimiento de la escala bioestratigráfica estándar de conodontos para el Devónico Inferior (ValenzuelaRíos, 1994a, b, 1999; Valenzuela-Ríos \& Murphy, 1997). En este sentido, los trabajos iniciales realizados en el área, principalmente por el Dr. Valenzuela-Ríos (ver, entre otros, García-López et al., 1990; Valenzuela-Ríos, 1990, 1994a y b, 1999, 2001, 2002; Valenzuela-Ríos \& Murphy, 1997; Sanz-López et al., 1999; Martínez-Pérez \& Valenzuela-
Ríos, 2005; Valenzuela-Ríos et al., 2005), han demostrado que el registro de conodontos pirenaicos es relativamente rico y abundante. Este registro está caracterizado tanto por taxones cosmopolitas, frecuentemente registrados en ambientes de aguas profundas (facies hercínica), como por aquellos taxones considerados endémicos y habitualmente encontrados en ambientes de aguas más someras (facies renana). Esta excepcional circunstancia hace que los Pirineos sean un área clave y de gran importancia para la bioestratigrafía del Devónico Inferior, ya que permite un control y comparación directa entre ambos tipos de ambientes, y por lo tanto realizar precisas correlaciones entre regiones que presentan importantes diferencias de facies (Valenzuela-Ríos, 1994a). 
Recientemente, el equipo del Devónico de la Universitat de València ha comenzado un estudio exhaustivo de los materiales de edad Emsiense del Pirineo Central Español, los cuales han proporcionado una importante sucesión de faunas de conodontos. Destacan diversos representantes del género Polygnathus Hinde, 1879, incluyendo especies con un alto valor bioestratigráfico como Po. pireneae Boersma, 1973, Po. kitabicus Yolkin et al., 1994, Po. excavatus Carls \& Gandl, 1969 o Po. nothoperbonus Mawson, 1987 (Valenzuela-Ríos, 1994a, 2001; MartínezPérez \& Valenzuela-Ríos, 2005). Junto a estos taxones, es remarcable la presencia de una abundante y diversificada asociación de especies del género Icriodus Branson \& Mehl, 1938, género que en este trabajo se utiliza en su sentido más amplio, entre las que destacan Ic. curvicauda Carls \& Gandl, 1969, Ic. celtibericus Carls \& Gandl, 1969, Ic. bilatericrescens Ziegler, 1956 o Ic. gracilis Bultynck, 1985; incluyendo también abundantes ejemplares de una nueva especie del mismo género. Está asociación de especies de Icriodus, hallada en los mismos estratos que importantes indicadores bioestratigráficos del género $\mathrm{Po}$ lygnathus, es de gran importancia, ya que permite el uso de estas especies de Icriodus como indicadores bioestratigráficos alternativos en otras regiones donde los taxones índices habituales son escasos o están ausentes (ver, por ejemplo, Sweet, 1988; Bultynck, 2003; Slavík, 2004a y b).

Según lo anteriormente expuesto, el propósito de este trabajo es describir una nueva especie de Icriodus hallada en los materiales del Devónico Inferior de los Pirineos Centrales Españoles, indicar su posición estratigráfica, y comentar brevemente las implicaciones de esta nueva especie en la diversificación del grupo durante el Praguiense tardío y el Emsiense inferior.

\section{PROCEDENCIA Y DEPÓSITO DEL MATERIAL}

El material aquí estudiado procede de cinco secciones levantadas en los estratos de edad Praguiense y Emsiense inferior del Pirineo Central Español. Estas cinco secciones son, de oeste a este (ver Fig. 1): las secciones Isábena-1 (Isb-1) y Baliera-6 (Bal-6), situadas en las cabeceras de los ríos Isábena y Baliera respectivamente; las secciones de La Guardia d'Àres, LGA$\mathrm{X}$ y LGA-XI, situadas en las proximidades de esta localidad; $\mathrm{y}$ la sección Villech-IA (Vi-IA), cerca de la aldea de Villech (ver Valenzuela-Ríos, 1994a, 2001, y Martinez-Pérez, 2010, para una descripción detallada de la situación geográfica de las secciones).

Las cinco secciones se hallan exclusivamente dentro del Área de Facies Sur de Mey (1967), la cual, se encuentra a su vez subdividida en otras unidades de menor rango o Subfacies: las Subfacies Sierra Negra, Baliera, Renanué y Compte (Mey, 1968; Habermehl, 1970; Hartevelt, 1970; Boersma, 1973; Valenzuela-Ríos \& Liao, 2006). Dentro de las Subfacies Baliera (Mey, 1968) se han levantado las secciones Isb-1 y Bal-6 (Fig. 1) en el Miembro Llaviero de la Formación Basibé. Esta uni- dad litológica está caracterizada por calizas organógenas de color negro azulado, tableadas y algo dolomitizadas, con algunos tramos de calizas masivas (ver Valenzuela-Ríos, 1994a, 2001, para una descripción detallada de estas secciones). Las secciones LGA-X, LGA-XI y Vi-IA han sido descritas dentro de las Subfacies Compte (Hartevelt, 1970) (Fig. 1), en niveles de la parte superior de la Formación Castanesa, caracterizada por calizas tableadas de espesor centimétrico a decimétrico de color gris-azulado con algunas intercalaciones milimétricas margosas, y el Miembro Capas de Castells de la Formación Villech, caracterizado por una sucesión de calizas tableadas arcillosas de color marrón-ocre con abundantes intercalaciones margosas del mismo color (ver Martínez-Pérez, 2010, para una descripción más detallada de las secciones). Todas las secciones fueron muestreadas exhaustivamente para el estudio de conodontos, con un total de 179 muestras y peso medio por muestra de unos 6,5 $\mathrm{kg}$, las cuales fueron procesadas con ácido fórmico al 7\% y lavadas posteriormente por decantación. Todos los ejemplares recuperados se encuentran depositados en el Museo de Geología de la Universitat de València bajo las siglas MGUV seguido de su correspondiente número de registro.

\section{SISTEMÁTICA PALEONTOLÓGICA}

Únicamente se han descrito los elementos "I" según Sweet (1981, 1988), utilizándose para ello los términos en castellano propuestos por García-López (1984). En el apartado del material de estudio se han utilizado una serie de abreviaturas para indicar la sección, el nivel de procedencia del material y la cantidad de ejemplares identificados. Así, el número siguiente al símbolo de la sección (ver arriba) separado por una " " señala el nivel de procedencia de la muestra, y los números entre paréntesis corresponden al número de ejemplares.

Clase CONODONTA Eichenberg, 1930 Orden PRIONIODONTIDA Dzik, 1976

Familia Icriodontidae Müller \& Müller, 1957

Género Icriodus Branson \& Mehl, 1938

Especie tipo: Icriodus expansus Branson \& Mehl, 1938.

\section{Icriodus riosi $\mathrm{n} . \mathrm{sp}$.}

Fig. 2

Derivatio nominis: Especie dedicada al Dr. José Ignacio Valenzuela Ríos, por su gran aportación al conocimiento de los conodontos del Devónico de los Pirineos Centrales Españoles.

Holotipo: MGUV-21.182 ejemplar representado en la figura $2 \mathrm{f}$. Locus typicus: Sección Baliera 6, levantada en el Mbr. Llaviero de la Fm. Basibé en el área del valle del Baliera (Provincia de Huesca, Pirineos Centrales Españoles) (ver Valenzuela-Ríos, 1994a, 2001, y Martínez-Pérez, 2010, para una descripción detallada de la sección).

Stratum typicum: Capa 49.

Paratipos: MGUV-21.162, MGUV-21.163, MGUV-21.168, MGUV-21.170, y MGUV-21.202 (Figs. 2a-e). 
Material: 39 ejemplares de los siguientes niveles: Isb-1/7c(1), Isb-1/8(1), Isb-1/10M(1), Isb-1/11(1), Bal-6/23(4), Bal-6/26(2), Bal-6/49(10), Bal-6/50(1), LGA-X/2(2), LGA-X/4(3), LGA$\mathrm{X} / 16 \mathrm{C}(8), \mathrm{LGA}-\mathrm{X} / 42(1)$, LGA-XI/2a(2), LGA-XI/12(1), ViIA/24(1). Material figurado MGUV-21.162, MGUV-21.163, MGUV-21.168, MGUV-21.170, MGUV-21.182 y MGUV-21.202. Resto del material MGUV-21.164, MGUV-21.168, MGUV21.169, MGUV-21.171 a MGUV-21.181, MGUV-21.183 a MGUV-21.188, MGUV-21.190 a MGUV-21.192, MGUV-21.197, MGUV-21.198, MGUV-21.200 a MGUV-21.203, MGUV-21.205, MGUV-21.290, MGUV-21.291 y MGUV-21.297.

Diagnosis: basada en elementos "I"; especie de Icriodus con una plataforma alargada, presentando bordes rectos sin una constricción posterior. Proceso posterior externo corto, formando con el eje principal del elemento un ángulo de entre $100^{\circ}$ y $130^{\circ}$. Cavidad basal estrecha y profunda expandiéndose ligeramente en su parte posterior.

Based on "I" elements; species of Icriodus with a long and straight platform without a posterior constriction of the spindle. The outer lateral process is short and forms an angle of $100^{\circ}$ to $130^{\circ}$ with the main axis of the element. Narrow and deep basal cavity, which is slightly expanded in its posterior part.

Descripción: El cuerpo principal es largo, recto y estrecho, produciéndose un estrechamiento del mismo hacia el extremo anterior, lo que le confiere al elemento una morfología lanceolada. Carena anterior recta o ligeramente curvada hacia el interior, formada, en la mayoría de los ejemplares, por entre 6 y 9 dentículos cónicos bien individualizados, algo comprimidos lateralmente y conectados por puentes longitudinales, desarrollándose en algunos ejemplares un dentículo aislado en el extremo anterior del elemento. La carena posterior está formada por 2-3 dentículos comprimidos lateralmente, de aspecto triangular en vista lateral y de una altura similar a los dentículos de la carena anterior, siendo el dentículo distal él de mayor tamaño. Cuando poseen tres dentículos, casi siempre el proximal es de menor tamaño y está fusionado en su base con el último dentículo de la carena anterior.

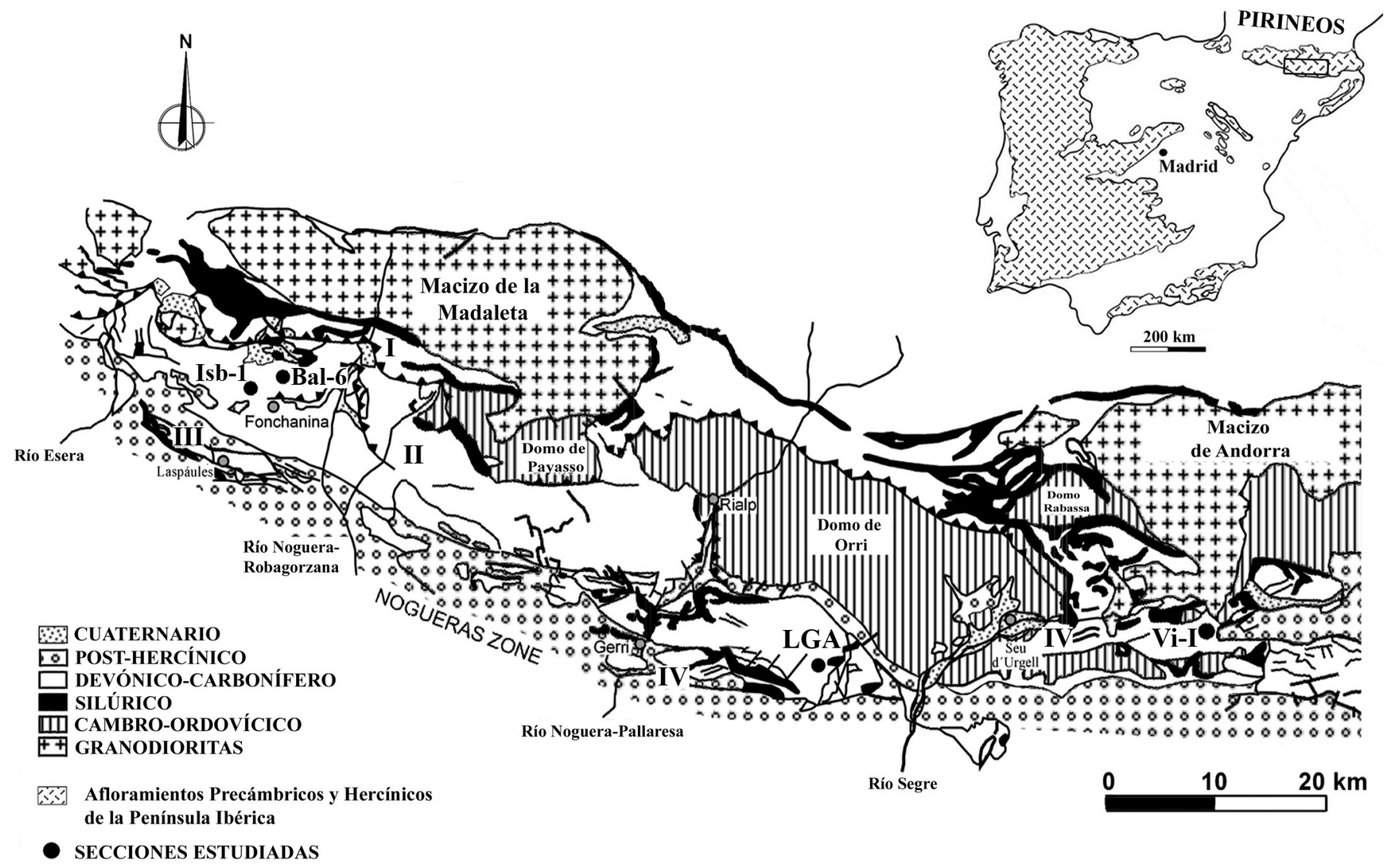

Figura 1. Esquema geológico del área de estudio, con indicación de las cuatro subfacies del área de Facies Sur (Mey, 1967): I) Subfacies Sierra Negra, II) Subfacies Baliera, III) Subfacies Renanué y IV) Subfacies Compte; junto con la situación geográfica de las secciones estudiadas: Isábena-1 (Isb-1), Baliera-6 (Bal-6), secciones de la Guardia d’Àres (LGA) y VillechIA (Vi-IA). Modificado de Valenzuela-Ríos (1994a).

Geological scheme of the study area in the Central Spanish Pyrenees, indicating the four Subfacies areas contained in the Palaeozoic outcrops of the extensive Southern Facies-area of Mey (1967): I) Sierra Negra Subfacies, II) Baliera Subfacies, III) Renanué Subfacies and IV) Compte Subfacies; with the location of the five sections studied: Isábena-1 (Isb-1), Baliera 6 (Bal), the LGA sections (LGA-X and LGA-XI), and Villech-IA section (Vi-I). Modified from Valenzuela-Rios (1994a). 

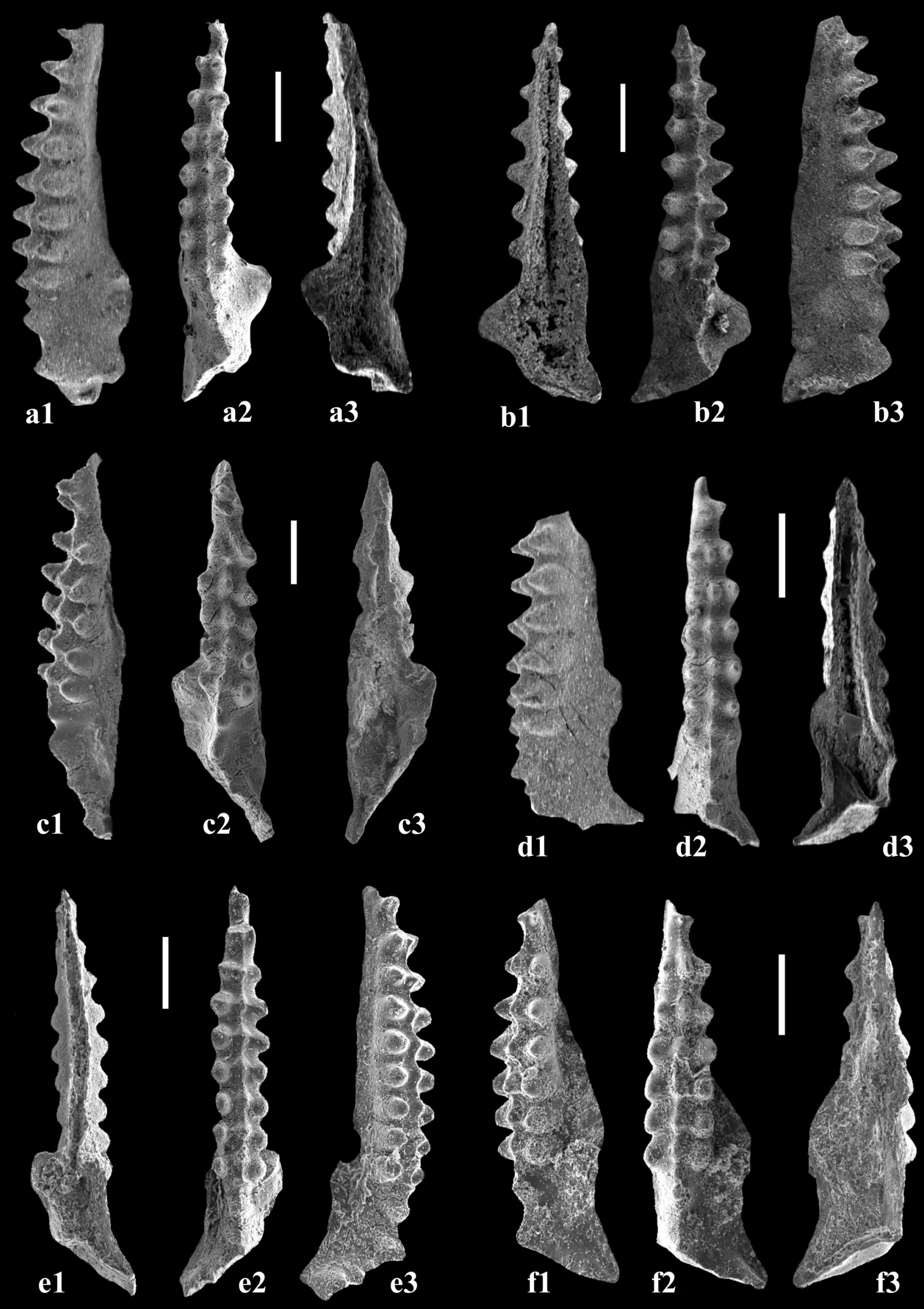
Las filas laterales están compuestas, en la mayoría de ejemplares estudiados, por 5-8 dentículos cónicos bien individualizados, de mayor tamaño que los de la fila media y alineados con ellos, excepto los de la mitad posterior, que están levemente retrasados.

La distancia entre las filas transversales es mayor en la región anterior, donde están separadas por profundos y anchos surcos transversales. Esta distancia se reduce en la mitad posterior, donde la separación entre filas es aproximadamente igual.

Proceso posterior externo corto, formando un ángulo usualmente de entre $100^{\circ}$ y $130^{\circ}$ con respecto a la fila media. Este proceso puede estar ornamentado por 2-3 dentículos comprimidos lateralmente, o por una fina cresta que recorre el proceso longitudinalmente.

Algunos ejemplares desarrollan un pequeño proceso anterior interno o espolón liso en el tercio posterior de la plataforma (ver, por ejemplo, Figs. 2, a2, b2, c2). Este espolón se encuentra dirigido anterolateralmente y es ligeramente redondeado, llegando su borde anterior hasta la última o penúltima fila del cuerpo principal.

La cavidad basal es estrecha y profunda, abriéndose en su tercio posterior, donde se desarrolla ligeramente la expansión labiada interna por debajo del proceso anterior. Surco anterior recto y profundo, el cual se une con el surco exterior con un ángulo aproximado de unos $130^{\circ}$

Comentarios: La presencia de un cuerpo principal con bordes rectos sin presentar una constricción de los mismos en su región posterior, es la característica diagnóstica más importante que posee Ic. riosi $\mathrm{n}$. sp.

Ic. riosi n. sp. presenta ciertas semejanzas con Ic. celtibericus Carls \& Gandl, 1969 compartiendo ambos un cuerpo largo y estrecho, y un proceso posterior corto que forma un ángulo mayor de $90^{\circ}$ con el eje principal del elemento (Figs. 3c y 3d). Sin embargo, Ic. celtibericus presenta una clara constricción en la región posterior del cuerpo principal (Figs. 3c1 y 3d1), constricción que está ausente en Ic. riosi n. sp. Además, Ic. riosi n. sp. se diferencia de otros taxones próximos, como Ic. curvicauda Carls \& Gandl, 1969, por el claro ángulo que forma el proceso posterior con el eje principal del elemento, ya que en Ic. curvicauda, el proceso posterior externo se prolonga como una continuación de la fila media sin llegar a formar un claro ángulo con ella (Figs. $3 a 2$ y 3b1). Asimismo, al igual que ocurría con Ic. celtibericus, Ic. curvicauda presenta una clara constricción en la región posterior del cuerpo principal, característica que también la diferencia de $I c$. riosi n. sp.

Igualmente, Ic. riosi n. sp. se distingue claramente del grupo de gracilis-bilatericrescens porque éstos últimos presentan un mayor número de filas transversales y por el mayor desarrollo de sus procesos laterales, uno posterior externo y otro anterior interno, formando entre ellos ángulos próximos a $180^{\circ}$ en Ic. bilatericrescens Ziegler, 1956 (Fig. 3f1) y aun mayores en Ic. gracilis Bultynck, 1985 (Fig. 3e2). Contrariamente, Ic. riosi n. sp. presenta unos procesos laterales muy poco desarrollados (estando en muchos casos el proceso anterior interno o espolón ausente) formando entre ellos ángulos claramente menores de $180^{\circ}$.

Por otro lado, debido al grado de desarrollo de la parte posterior de la plataforma, tanto por su cavidad basal como por el estado incipiente de desarrollo de sus procesos, Ic. riosi $\mathrm{n}$. sp. probablemente está filogenéticamente relacionado con las especies del grupo curvicauda-celtibericus, más concretamente con Ic. celtibericus según la forma y estado de desarrollo de su proceso posterior externo.

Distribución estratigráfica y geográfica: La distribución de Ic. riosi n. sp. comprende desde la Zona pireneae del Praguiense hasta el Emsiense inferior tardío (parte temprana de la Zona nothoperbonus; Fig. 4). Hasta el presente, se ha encontrado únicamente en los Pirineos.

\section{DISCUSIÓN}

El estudio de 5 secciones (Isb-1, Bal-6, LGA-X, LGAXI y Vi-IA) de edad Praguiense superior y Emsiense inferior en los Pirineos Centrales Españoles ha proporcionado una importante asociación de conodontos entre los que podemos encontrar diferentes especies de los géneros Polygnathus e Icriodus, todas ellas con un alto valor bioestratigráfico. El aumento en el número de especies conocidas

Figura 2. a: Icriodus riosi n. sp., paratipo, MGUV-21.162; a1, vista lateral; a2, vista oral; a3, vista aboral; nivel de procedencia LGA-X/16C. b: Icriodus riosi n. sp., paratipo, MGUV-21.163; b1, vista aboral; b2, vista oral; b3, vista lateral; nivel de procedencia LGA-X/16C. c: Icriodus riosi n. sp., paratipo, MGUV-21.170; c1, vista lateral; c2, vista oral; c3, vista aboral; nivel de procedencia Isb-1/10M. d: Icriodus riosi n. sp., paratipo, MGUV-21.202; d1, vista lateral; d2, vista oral; d3, vista aboral; nivel de procedencia LGA-X/16C. e: Icriodus riosi n. sp., paratipo, MGUV-21.168; e1, vista aboral; e2, vista oral; e3, vista lateral; nivel de procedencia Bal-6/49. f: Icriodus riosi n. sp., holotipo, MGUV-21.182; f1, vista lateral; f2, vista oral; f3, vista aboral; nivel de procedencia Bal-6/49. Todas las barras de escalas representan $200 \mu \mathrm{m}$. a: Icriodus riosi n. sp., paratype, $M G U V-21.162$; a1, lateral view; a2, oral view; a3, aboral view; LGA-X/16C. b: Icriodus riosi $n$. sp., paratype, $M G U V-21.163$; b1, aboral view; b2, oral view; b3, lateral view; LGA-X/16C. c: Icriodus riosi n. sp., paratype, MGUV-21.170; c1, lateral view; c2, oral view; c3, aboral view; Isb-1/10M. d: Icriodus riosi $n$. sp., paratype, $M G U V-21.202$; d1, lateral view; d2, oral view; d3, aboral view; LGA-X/16C. e: Icriodus riosi $n$. sp., paratype, MGUV-21.168; e1, aboral view; e2, oral view; e3, lateral view; Bal-6/49. f: Icriodus riosi n. sp., holotype, MGUV-21.182; f1, lateral view; f2, oral view; f3, aboral view; Bal-6/49. All scale bars represent $200 \mu \mathrm{m}$. 


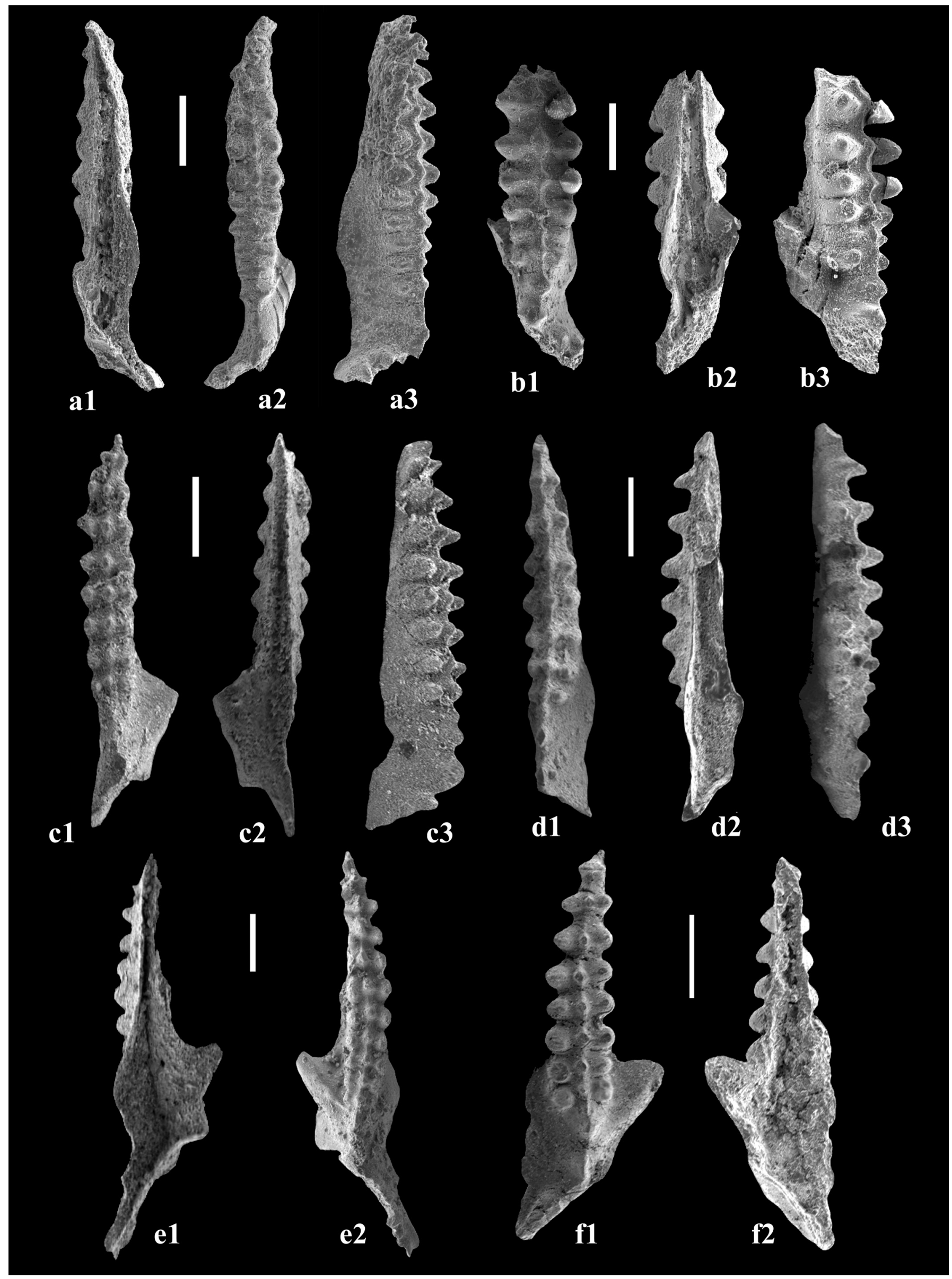




\begin{tabular}{|c|c|c|c|c|c|}
\hline Piso & Praguiense & \multicolumn{4}{|c|}{ Emsiense } \\
\hline \multirow{2}{*}{$\begin{array}{l}\text { Taxones de Zonas de } \\
\text { conodontos } \\
\text { conodos }\end{array}$} & \multirow{2}{*}{ pireneae } & \multirow{2}{*}{ kitabicus } & \multicolumn{2}{|c|}{ excavatus } & \multirow{2}{*}{ nothoperbonus } \\
\hline & & & inferior & media & \\
\hline \multicolumn{6}{|l|}{ Ic. curvicauda } \\
\hline \multicolumn{6}{|l|}{ Ic. celtibericus } \\
\hline \multicolumn{6}{|l|}{ IC. riosi n. sp. } \\
\hline Ic. gracilis & & & & & \\
\hline Ic. bilatericrescens & & & & & \\
\hline
\end{tabular}

Figura 4. Esquema de la distribución estratigráfica de los principales taxones de Icriodus identificados alrededor del actual límite Praguiense/Emsiense (en el sentido de la SDS) y Emsiense inferior basada en los registros de conodontos encontrados en las diferentes secciones pirenaicas estudiadas (esquema bioestratigráfico basado en Martínez-Pérez, 2010).

Scheme of the stratigraphical distribution of the main Icriodus species around the present Pragian/Emsian boundary (sensu SDS) and lower Emsian based on the conodont record found in the different studied pyrenean sections (biostratigraphical scheme based on Martinez-Pérez, 2010).

durante el Praguisene final y Emsiense temprano (en el sentido tradicional alemán de Carls, 1987), ya había sido descrito para el género Polygnathus. Motivo por el cual, algunos autores proponen la existencia de una radiación evolutiva de este género durante este intervalo temporal (Yolkin et al., 1994, 2008; Bardashev et al., 2002). Sin embargo, es importante destacar que, hasta ahora, esta radiación únicamente ha sido bien documentada en el área de la sección del actual Global Stratotype Section and Point (GSSP) del Emsiense en Asia Central (Yolkin et al., 1994, 2008) y en los Pirineos (Martínez-Pérez, 2010). No obstante, la aparición, con estos taxones de Polygnathus, de una abundante y diversificada asociación de especies de Icriodus, como Ic. curvicadua, Ic. celtibericus, Ic. bilatericrescens o Ic. gracilis, sumada a la nueva especie aquí descrita, Ic. riosi, parecen indicar que las innovaciones que se produjeron durante ese momento, no afectaron únicamente a las especies del género Polygnathus, sino que el género Icriodus, también experimentó un proceso de radiación evolutiva como lo evidencian la diversidad de los taxones encontrados, cuyas distribuciones estratigráficas se muestran en la figura 4. Ic. riosi n. sp, como ya se ha comentado, aparece ligeramente por debajo del comienzo del actual, y momentáneamente oficial (ver Carls et al., 2008), límite kitabicus (actual límite Praguiense/Emsiense) llegando sus últimos registros a la parte más inferior de la Zona nothoperbonus (Emsiense inferior tardío) (Fig. 4).

Así pues, la presencia conjunta en las mismas secciones de diversas especies de Icriodus, incluida la nueva especie aquí descrita, con importantes bioindicadores del genéro Polygnathus, los cuales son la base de la actual biozonación del Emsiense (Klapper \& Johnson, 1975; Klapper, 1977; Weddige \& Ziegler, 1977; Lane \& Ormiston, 1979; Yolkin et al., 1994; Bardashev et al., 2002), convierte a los Pirineos en un área clave para el estudio de este Piso. Según esto, con la aparición conjunta de diferentes especies de ambos géneros, es importante señalar que las especies de Icriodus podrían presentar, en estos casos, un alto potencial para correlaciones suprarregionales, especialmente en Ibero-Armórica, donde se encuentran asociadas a ricas

Figura 3. a: Icriodus curvicauda Carls \& Gandl, 1969, MGUV-21.219; a1, vista aboral; a2, vista oral; a3, vista lateral; nivel de procedencia Bal-6/49. b: Icriodus curvicauda Carls \& Gandl, 1969, MGUV-21.236; b1, vista oral; b2, vista aboral; b3, vista lateral; nivel de procedencia Bal-6/49. c: Icriodus celtibericus Carls \& Gandl, 1969, MGUV-21.195; c1, vista oral; c2, vista aboral;c3, vista lateral; nivel de procedencia LGA-X/4. d: Icriodus celtibericus Carls \& Gandl, 1969, MGUV21.206; d1, vista oral; d2, vista aboral; d3, vista lateral; nivel de procedencia LGA-XI/2b. e: Icriodus gracilis Bultynck, 1985, MGUV-21.239; e1, vista aboral; e2, vista oral; nivel de procedencia LGA-X/8. f: Icriodus bilatericrescens Ziegler, 1956, MGUV-21.148; f1, vista oral; f2, vista aboral; nivel de procedencia Bal-6/49. Todas las barras de escalas represen$\tan 200 \mu \mathrm{m}$.

a. Icriodus curvicauda Carls \& Gandl, 1969, MGUV-21.219; a1, aboral view; a2, oral view; a3, lateral view; Bal-6/49. b: Icriodus curvicauda Carls \& Gandl, 1969, MGUV-21.236; b1, oral view; b2, aboral view; b3, lateral view; Bal-6/49. c: Icriodus celtibericus Carls \& Gandl, 1969, MGUV-21.195; c1, oral view; c2, aboral view; c3, lateral view; LGA-X/4. $\boldsymbol{d}$ : Icriodus celtibericus Carls \& Gandl, 1969, MGUV-21.206; d1, oral view; d2, aboral view; d3, lateral view; LGA-XI/2b. $\boldsymbol{e}$ : Icriodus gracilis Bultynck, 1985, MGUV-21.239; e1, aboral view; e2, oral view; LGA-X/8. $\boldsymbol{f}$ : Icriodus bilatericrescens Ziegler, 1956, MGUV-21.148; f1, oral view; f2, aboral view; Bal-6/49. All scale bars represent $200 \mu \mathrm{m}$. 
shelly faunas de aguas someras. Por otro lado, estas mismas especies podrían ser utilizadas como indicadores bioestratigráficos alternativos, cuando en otras regiones los taxones índices habituales son escasos o están ausentes, como ya han propuesto diversos autores (ver, por ejemplo, Sweet, 1988; Bultynck, 2003; Slavík, 2004a y b).

Por último, destacar que el presente estudio permite aumentar el conocimiento sobre las faunas de conodontos del Devónico Inferior de los Pirineos Centrales Españoles, lo que incidirá a su vez en una mejor comprensión de la distribución a nivel regional y suprarregional de las faunas de conodontos durante el Praguiense tardío y el Emsiense inferior. Tales datos son de gran importancia para, por ejemplo, la discusión de la actual biozonación del Emsiense y para la redefinición de su límite inferior, temas que se encuentran actualmente en discusión (ver un resumen del estado actual del tema en Carls et al., 2008).

\section{AGRADECIMIENTOS}

Quisiera agradecer y dedicar este trabajo al Dr. José I. Valenzuela-Ríos (Universitat de València), por su apoyo y ayuda, en este y otros proyectos. También agradezco las correcciones y comentarios del Dr. Peter Carls (Technische Universität Braunschweig) y la Dr. Susana García-López (Universidad de Oviedo), así como las del editor Dr. Rodolfo Gozalo (Universitat de València) que han permitido mejorar notablemente este trabajo. A Teresa (J-C. Liao, Universitat de València) su ayuda en el campo; y a Pilar Navas-Parejo (Universidad de Granada) y Pablo Plasencia (Universitat de València) por sus comentarios sobre una primera versión del presente manuscrito. Este trabajo es una contribución al proyecto PICG n ${ }^{\circ} 499$ : "Devonian land-sea interaction: evolution of ecosystems and climate" (DEVEC).

\section{REFERENCIAS BIBLIOGRÁFICAS}

Bardashev, I.A., Weddige, K. \& Ziegler, W. 2002. The phylomorphogenesis of some Early Devonian platform conodonts. Senckenbergiana lethaea, 82, 375-451.

Boersma, K.T. 1973. Devonian and lower Carboniferous conodont biostratigraphy, Central Spanish Pyrenees. Leidse Geologische Mededelingen, 49, 303-377.

Branson, E.B. \& Mehl, M.G. 1938. The conodont genus Icriodus and its stratigraphic distribution. Journal of Paleontology, 12, 156-166.

Bultynck, P. 1985. Lower Devonian (Emsian)-Middle Devonian (Eifelian and lowermost Givetian) conodont successions from the Ma'der and the Tafilalt, southern Morocco. Courier Forschungsinstitut Senckenberg, 75, 261-286.

Bultynck, P. 2003. Devonian Icriodontidae: biostratigraphy, classification and remarks on paleoecology and dispersal. Revista Española de Micropaleontología, 35, 295-314.

Carls, P. 1987. Ein Vorschlag zur biostratigraphischen Redefinition der Grenze Gedinnium/Siegenium und benachbarter
Unter-Stufen. Courier Forschungsinstitut Senckenberg, 92, 77-121.

Carls, P. \& Gandl, J. 1969. Stratigraphie und Conodonten des Unter-Devons der Östlichen Iberischen Ketten (NESpanien). Neues Jahrbuch Geologie und Paläontologie, Abhandlungen, 132, 155-218.

Carls, P., Slavík, L. \& Valenzuela-Ríos, J.I. 2008. Comments on the GSSP for the basal Emsian stage boundary: the need for its redefinition. Bulletin of Geosciences, $\mathbf{8 3}$, 383-390.

Dzik, J. 1976. Remarks on the evolution of Ordovician conodonts. Acta Palaeontologica Polonica, 21, 395-455.

Eichenberg, W. 1930. Conodonten aus dem Culm des Harzes. Paläontologische Zeitschrift, 12, 177-182.

García-López, S. 1984. Terminología morfológica de conodontos compuestos y de plataforma. Revista de Biología de la Universidad de Oviedo, 2, 95-105.

García-López, S., García-Sansegundo, J. \& Arbizu, M. 1990. Datos estratigráficos y paleontológicos de la sucesión devónica del área del río Baliera (Zona Axial, Pirineos centrales españoles). Geogaceta, 7, 33-35.

Habermehl, M.A. 1970. Depositional history and diagenesis of quartz-sand bars and lime-mud environments in the Devonian Basibé Formation (Central Pyrenees, Spain). Leidse Geologische Mededelingen, 46, 1-55.

Hartevelt, J.J.A. 1970. Geology of the upper Segre and Valira valleys, Central Pyrenees, Andorra/Spain. Leidse Geologische Mededelingen, 45, 167-236.

Hinde, G.J. 1879. On conodonts from the Chazy and Cincinnatti Group of the Cambro-Silurian, and from the Hamilton and Genesee-Shale division of the Devonian, in Canada and the United States. Geological Society of London, Quarterly Journal, 35, 351-369.

Klapper, G. 1977. Lower and Middle Devonian conodont sequence in central Nevada. In: Western North America: Devonian (eds. M.A. Murphy, W.B.N. Berry \& C.A. Sandberg). Riverside Campus Museum Contribution, University of California, 4, 33-54.

Klapper, G. \& Johnson, D.B. 1975. Sequence in the conodont genus Polygnathus in the lower Devonian at Lone Mountain, Nevada. Geologica et Palaeontologica, 9, 65-97.

Lane, H.R. \& Ormiston, A.R. 1979. Siluro-Devonian biostratigraphy of the Salmontrout River Area, east-central Alaska. Geologica et Palaeontologica, 13, 39-96.

Martinez-Pérez, C. 2010. Conodontos del Emsiense (Devónico Inferior) del Pirineo Central Español. Tesis doctoral, Universitat de València, $376 \mathrm{pp}$. (inédita).

Martínez-Pérez, C. \& Valenzuela-Ríos, J.I. 2005. Conodontos del Límite Praguiense/Emsiense (Devónico Inferior) en la sección Isábena 1 (Huesca, Pirineo Aragonés). In: Miscelánea Paleontológica (eds. G. Meléndez, C. MartínezPérez, S. Ros, H. Botella \& P. Plasencia). Publicaciones del Seminario de Paleontología de Zaragoza, 6, 287-319.

Mawson, R. 1987. Early Devonian Conodont faunas from Buchan' Victoria, and Bindi, Australia. Palaeontology, 30, 251-297.

Mey, P.H.W. 1967. Evolution of the Pyrenean Basin during the Late Palaeozoic. International Symposium on the Devonian System, Calgary, 2, 1157-1166. 
Mey, P.H.W. 1968. The geology of the upper Ribagorzana and Tor Valleys, Central Pyrenees, Spain sheet 8, 1:50000. Leidse Geologische Mededelingen, 41, 229-292.

Müller, K.J. \& Müller, E.M. 1957. Early Upper Devonian (Independence) conodonts from Iowa, Part 1. Journal of Paleontology, 31, 1069-1108.

Sanz-López, J., Valenzuela-Ríos, J.I., García-López, S., Gil Peña, I. \& Robador, A. 1999. Nota preliminar sobre la estratigrafía y el contenido en conodontos del PrídolíLochkoviense inferior en la unidad de els Castells (Pirineo central). Temas Geológico-Mineros ITGE, 26, 638-642.

Slavík, L. 2004a. A new conodont zonation of the Pragian in the stratotype area (Barrandian, central Bohemia). Newsletters on Stratigraphy, 40, 39-71.

Slavík, L. 2004b. The Pragian-Emsian conodont successions of the Barrandian area: search of an alternative to the GSSP polygnathid-based correlation concept. Geobios, 37, 454-470.

Sweet, W.C. 1981. Glossary of morphological and structural terms for conodont elements and apparatuses. In: Treatise on Invertebrate Paleontology, Part W, Miscellanea Supplement 2, Conodonta. Geological Society of America, 60-67.

Sweet, W.C. 1988. The conodonta. Morphology, Taxonomy, Paleoecology and Evolutionary History of a Long-Extinct Animal Phylum. Oxford Monographs on Geology and Geophysics, 10, $212 \mathrm{pp}$.

Valenzuela-Ríos, J.I. 1990. Lochkovian conodonts and stratigraphy at Gerri de la Sal (Pyrenees). Courier Forschungsinstitut Senckenberg, 118, 53-56

Valenzuela-Ríos, J.I. 1994a. Conodontos del Lochkoviense y Praguiense (Devónico Inferior) del Pirineo Central español. Memorias del Museo Paleontológico de la Universidad de Zaragoza, 5, 178 pp.

Valenzuela-Ríos, J.I. 1994b. The Lower Devonian conodont Pedavis pesavis and the pesavis zone. Lethaia, 27, 199207.

Valenzuela-Ríos, J.I. 1999. Ancyrodelloides sequeirosi, un nuevo conodonto del Lochkoviense Medio (Dévonico Inferior) de los Pirineos Orientales Españoles. In: 25 años de Paleontología Aragonesa. Homenaje al profesor Leandro Sequeiros (eds. J. A. Gámez Vinatend \& E. Liñan). Institución Fernando el Católico, Zaragoza, 247-253

Valenzuela-Ríos, J.I. 2001. Polygnátidos primitivos en los Pirineos; un argumento más en contra del actual límite Praguiense/Emsiense (Devónico Inferior) en Zinzilbán (Uzbekistán). In: Los fósiles y la Paleogeografía (eds. G. Meléndez, Z. Herrera, G. Delvene \& B. Azanza). Pu- blicaciones del Seminario de Paleontología de Zaragoza, 5, 571-577

Valenzuela-Ríos, J.I. 2002. Lochkovian and Pragian conodonts from Segre 1 (Central Spanish Pyrenees). In: Palaeozoic conodonts from northern Spain. Eighth International Conodont Symposium held in Europe (eds. S. García-López \& F. Bastida). Publicaciones del Instituto Geológico y Minero de España, Serie: Cuadernos del Museo Geominero, Madrid, 1, 403-417.

Valenzuela-Ríos, J.I. \& Liao, J-C. 2006. Annotations to Devonian Correlation Table, R 357-360 di-ds 06: Spanish Central Pyrenees, Southern part. Senckenbergiana Lethaea, 86, 105-107.

Valenzuela-Rios, J.I. \& Murphy, M.A. 1997. A new zonation of middle Lochkovian (Lower Devonian) conodonts and evolution of Flajsella n. gen. (Conodonta). Geological Society of America Special Paper, 321, 131-144.

Valenzuela-Ríos, J.I., Liao, J-C., Martínez-Pérez, C., Castelló, V. \& Botella, H. 2005. Datos preliminares sobre los conodontos y restos de peces del Lochkoviense (Devónico Inferior) de Compte-I (Valle del Noguera Pallaresa, Pirineos de la Corona de Aragón). In: La cooperación Internacional en la Paleontología española. Homenaje al Profesor Peter Carls (eds. J.A. Gámez Vintaned, E. Liñán \& J.I. Valenzuela-Ríos). Institución Fernando el Católico, Zaragoza, 123-136.

Weddige, K. \& Ziegler, W. 1977. Correlation of Lower/Middle Devonian boundary beds. Newsletter on Stratigraphy, 6 , 67-84

Yolkin, E.A., Weddige, K., Izokh, N.G. \& Erina, M.V. 1994. New Emsian conodont zonation (Lower Devonian). Courier Forschungsinstitut Senckenberg, 168, 139-157.

Yolkin, E.A., Weddige, K., Izokh, N.G. \& Erina, M.V. 2008. Pragian-Early Emsian Polygnathids from Kitab Reserve sequences: their origin, main evolutionary stock and species lineages. In: Devonian sequences of the Kitab Reserve area (eds. E.A. Yolkin, A.I. Kim \& J.A. Talent). International Conference "Global Alignments of Lower Devonian Carbonates and clastic sequences" (SDS/IGCP 499 Project joint field meeting), 92-97.

Ziegler, W. 1956. Unterdevonische Conodonten, insbesondere aus dem Schönauer und dem Zorgensis-Kalk. Notizblatt des Hessischen Landesamtes für Bodenforschung, 84, 93-106.

Manuscrito recibido: 7 de Septiembre, 2010 Manuscrito aceptado: 11 de Noviembre, 2010 